\title{
Immunogenetic Epidemiology of Dementia and Parkinson's Disease in 14 Continental European Countries: Shared Human Leukocyte Antigen (HLA) Profiles
}

\author{
Lisa M. James ${ }^{1,2,3}$, Apostolos P. Georgopoulos $1,2,3,4 *$ \\ 'The HLA Research Group, Brain Sciences Center, Department of Veterans Affairs Health Care System, Minneapolis, MN, 55417, USA \\ ${ }^{2}$ Department of Neuroscience, University of Minnesota Medical School, Minneapolis, MN 55455, USA \\ ${ }^{3}$ Department of Psychiatry, University of Minnesota Medical School, Minneapolis, MN 55455, USA \\ ${ }^{4}$ Department of Neurology, University of Minnesota Medical School, Minneapolis, MN 55455, USA
}

Article Info

\section{Article Notes}

Received: February 23, 2021

Accepted: April 14, 2021

\section{${ }^{*}$ Correspondence:}

${ }^{*}$ Dr. Apostolos P. Georgopoulos, Brain Sciences Center (11B), Minneapolis VAHCS, One Veterans Drive, Minneapolis, MN 55417, USA; Email: omega@umn.edu.

(c) 2021 Georgopoulos AP. This article is distributed under the terms of the Creative Commons Attribution 4.0 International License.

\section{Keywords}

Dementia; Parkinson's disease

Human leukocyte antigen

Immunity

Genetics

\section{ABSTRACT}

Human leukocyte antigen (HLA), which is critically involved in immune response to foreign antigens and in autoimmunity, has been implicated in dementia and Parkinson's disease. Here we report on the correlations between the population frequencies of 127 HLA Class I and II alleles and the population prevalence of dementia and Parkinson's disease in 14 Continental Western European countries, extending previous work ${ }^{1,2}$. We used these correlations to construct and compare HLA profiles for each disease ${ }^{3}$. We found that (a) the HLA profiles of the two diseases were significantly correlated across both HLA Class I and Class II alleles, (b) negative ("protective") HLA-disease correlations did not differ significantly for either HLA class, but (c) positive ("susceptibility") HLAdisease correlations were significantly higher in dementia than in Parkinson's disease for both HLA classes of alleles. These findings indicate that (a) dementia and Parkinson's disease share immunogenetic HLA-related mechanisms, (b) HLA-related protective mechanisms (presumably against pathogens) do not differ between the two diseases, but (c) HLA-related susceptibility mechanisms (presumably underlying autoimmunity) are significantly stronger in dementia than in Parkinson's disease.

\section{Introduction}

Nearly 50 million people worldwide are living with dementia or Parkinson's disease, the two most common neurodegenerative diseases, resulting in a considerable toll on affected individuals, caregivers, and society ${ }^{4,5}$. Despite decades of extensive investigation, the etiology of both conditions remains unknown, hampering intervention and prevention efforts; however, growing research points to the involvement of pathogens as potential causes ${ }^{6-8}$ and autoimmunity as pathogenic mechanisms that are triggered by, and subsequently contribute to, chronic neuroinflammation in both conditions ${ }^{9-13}$. In light of overlapping pathogen associations and autoimmune-related neuroinflammation in dementia and Parkinson's disease, and the known involvement of the Human Leukocyte Antigen (HLA) in pathogen elimination and autoimmunity, we sought in the present study to evaluate the correspondence between the HLA disease profiles in these two diseases as an indicator of immunogenetic overlap.

The HLA system is involved in both pathogen elimination (as a preventive/protective factor) and autoimmunity (as a susceptibility factor). Two main classes of HLA genes code for cell-surface glycoproteins that are critically involved in facilitating cellular and 
humoral immune system responses to foreign antigens derived from various pathogens. With respect to pathogen elimination, HLA Class I molecules (of the A, B, C genes) are expressed on nucleated cells and present intracellular antigen peptides to CD8+ cytotoxic T cells to signal cell destruction, thus eliminating infected cells. On the other hand, HLA Class II molecules (of the DR-, DQ- and DPgenes) are expressed on professional antigen-presenting cells (e.g., macrophages, dendritic cells) and present endocytosed extracellular antigen peptides to CD4+ T cells to promote B-cell mediated antibody production against the offending pathogens and adaptive immunity for the future. HLA molecules are coded in the Major Histocompatibility Complex (MHC) in chromosome 6. MHC is the most highly polymorphic region in the human genome resulting in considerable individual and population variation in HLA composition, reflecting the long evolutionary history of exposure to and dealing with elimination of, and ultimate protection from, various pathogens ${ }^{14,15}$. With respect to autoimmunity, both HLA Class I and Class II molecules are intimately involved in autoimmune disorders ${ }^{16}$.

Burgeoning evidence has demonstrated HLA

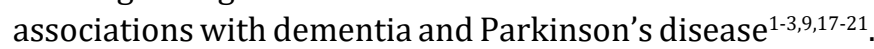
Using an across-countries population immunogenetic epidemiological approach ${ }^{22}$, we initially investigated associations between the population frequency of HLA Class II DRB1 alleles and dementia across 14 countries in Continental Western Europe ${ }^{1,2}$. This approach permits identification of an HLA profile wherein HLA alleles may presumably be characterized as protective alleles (negatively correlated with population prevalence of a disease) or susceptibility alleles (positively correlated with the population prevalence of a disease). With respect to HLA Class II DRB1 alleles, we recently found that the HLA profiles of dementia and Parkinson's disease for a small number of HLA alleles are very similar, suggesting that similar immunogenetic mechanisms may underlie the pathogenesis of these conditions ${ }^{3}$. Here we extend that line of research to evaluate the correspondence in the association of the population frequency of 127 HLA Class I and Class II alleles and the population prevalence of dementia and Parkinson's disease which will permit characterization of HLA-disease profiles. Our hypothesis is that, in part at least, these diseases are caused by pathogens which, if allowed sustained presence in the body, may inflict brain damage both directly (by killing cells and inflammation) and/or indirectly (by autoimmunity). Since the HLA system is involved both in pathogen elimination and autoimmunity, we focus on HLA association with disease prevalence as a "bird's eye view" to gain an insight into the involvement of pathogen elimination and autoimmunity in the pathogenesis of PD and dementia. The key point is that a pathogen elimination effect would be manifested as a negative correlation between disease prevalence and allele frequency, whereas, in contrast, autoimmunity would be manifested as a positive correlation.

\section{Materials and Methods}

\section{Prevalence of dementia and Parkinson's disease}

The population prevalence of dementia and Parkinson's disease was computed for each of the following 14 countries in Continental Western Europe: Austria, Belgium, Denmark, Finland, France, Germany, Greece, Italy, Netherlands, Portugal, Norway, Spain, Sweden, and Switzerland. Specifically, the total number of people with dementia or Parkinson's disease in each of the 14 Continental Western European countries as determined by the Global Burden of Disease study ${ }^{4,5}$ was divided by the total population of each country in 2016 (Population Reference Bureau) ${ }^{23}$ and expressed as a percentage. We have previously shown that life expectancy for these countries are virtually identical ${ }^{1}$; therefore, life expectancy was not included in the current analyses.

\section{HLA}

The frequencies of all reported HLA alleles of classical genes of Class I (A, B, C) and Class II (DPB1, DQB1, DRB1) for each of the 14 Continental Western European countries were retrieved from the website allelefrequencies.net (Estimation of Global Allele Frequencies ${ }^{24,25}$ ) on October 20, 2020. There was a total of 2746 entries of alleles from the 14 Continental Western European countries, comprising 844 distinct alleles. Of those, 127 alleles occurred in 9 or more countries and were used in further analyses. The distribution of those alleles to the HLA classes and their genes is given in Table 1 .

\section{Data analysis}

HLA profiles for Parkinson's disease and dementia were derived as described previously ${ }^{3}$. Briefly, the prevalences (fractions of total country population) of Parkinson's disease and dementia were natural-log transformed ${ }^{1-3}$ (see below) and the Pearson correlation coefficient, $r$, between disease prevalence and the population frequency of each one of the 127 HLA alleles above calculated and Fisher z-transformed ${ }^{26}$ to normalize their distribution:

$$
r^{\prime}=\operatorname{atanh}(r)
$$

The HLA disease profile consisted of 127 values of $r^{\prime}$. The association between the HLA profiles of Parkinson's disease and dementia was computed as the Pearson correlation between their HLA profiles. Since negative and

Table 1: Distribution of 127 HLA alleles analyzed to Class and Genes.

\begin{tabular}{|l|l|l|l|l|l|l|}
\hline & \multicolumn{3}{|c|}{ Class I (N= 69) } & \multicolumn{3}{c|}{ Class II (N = 58) } \\
\hline Gene & A & B & C & DPB1 & DQB1 & DRB1 \\
\hline Count & 20 & 36 & 13 & 15 & 14 & 29 \\
\hline
\end{tabular}


positive correlations $(-r,+r)$ between allele frequency and disease prevalence can be interpreted as indicating a protective or susceptibility effect of the allele on the disease, respectively, additional analyses were carried out on the counts of signed (negative, positive) correlations using two-way tables and associated statistics (chi-square test, Fisher's exact test, phi coefficient). Finally, the quantitative differences between Parkinson's disease and dementia with respect to protective (negative $r^{\prime}$ ) and susceptibility (positive $r^{\prime}$ ) alleles were assessed by a paired t-test in two groups, namely one on alleles protective to both diseases, and the other on susceptibility alleles for both diseases. Statistical analyses were performed using the IBM-SPSS package (IBM SPSS Statistics for Windows, Version 26.0, 64-bit edition. Armonk, NY: IBM Corp; 2019) and Intel FORTRAN (Microsoft Visual Studio Community Version 16.8.3; Intel FORTRAN Compiler 2021).

\section{Results}

HLA-disease profiles consist of correlations between allele frequency and disease prevalence, suitably Fisher z-transformed (Equation 1) to normalize their distribution for further analyses. We showed previously ${ }^{1}$ that dementia prevalence varies in an exponential fashion with allele frequency, such that the logarithm of disease prevalence is a linear function of allele frequency. Therefore, the correlation entered in a HLA-disease profile is that computed using the natural log-transformed disease prevalence and allele frequency. Two examples are illustrated in Figures 1 and 2, one for a presumed dementia protective allele (DRB1*04:01; Figure 1) and another for a presumed dementia susceptibility allele (DPB1*02:01; Figure 2).

\section{HLA profiles of Parkinson's disease and dementia}

The frequency distributions of alleles in HLA profiles for Parkinson's disease and dementia (Table 2) are shown in Figures 3 and 4, respectively. It can be seen that they are similar, with a broad overlap. The HLA profiles of the two diseases were positively and highly significantly correlated (Figure 5) $\left(\mathrm{r}=0.904, \mathrm{P}=6.4 \times 10^{-48}, \mathrm{~N}=127\right)$. This positive association extended across the two HLA classes (color

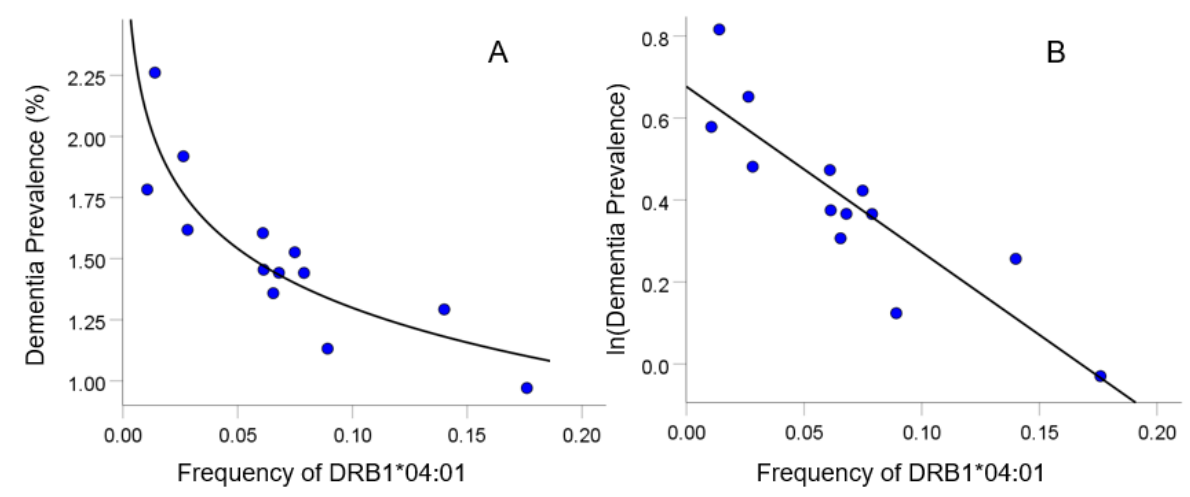

Figure 1. Example from a presumed protective HLA allele. Dementia prevalence for 13 CWE countries (for which DRB1*04:01 frequency was available) is plotted against the corresponding DRB1*04:01 allele frequency in the original (percentage) dementia prevalence scale (A) and its natural log transformed values (B). The fitted line is an exponential function (A) that becomes a linear function in the logtransformed prevalence scale (B). The statistics for the linear case are: Pearson correlation $r=-0.877, P<0.001 ; r^{\prime}=\operatorname{atanh}(r)=-1.363$.
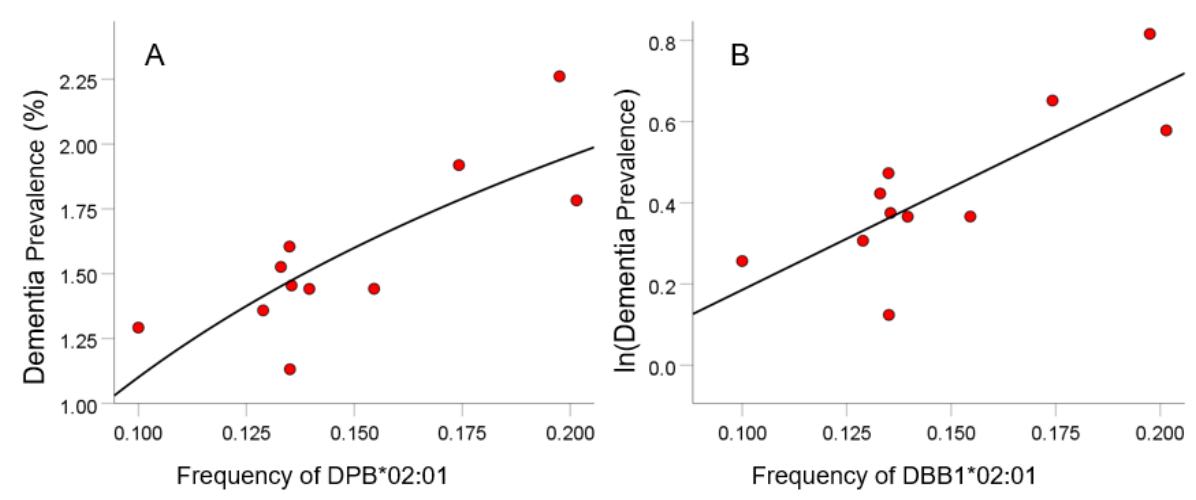

Figure 2. Example from a presumed susceptibility HLA allele. Dementia prevalence for 11 CWE countries (for which DPB1*02:01 frequency was available) is plotted against the corresponding DPB*02:01 allele frequency in the original (percentage) dementia prevalence scale (A) and its natural log transformed values (B). The fitted line is an exponential function (A) that becomes a linear function in the logtransformed prevalence scale (B). The statistics for the linear case are: Pearson correlation $r=-0.805, P=0.003 ; r^{\prime}=\operatorname{atanh}(r)=1.112$. 


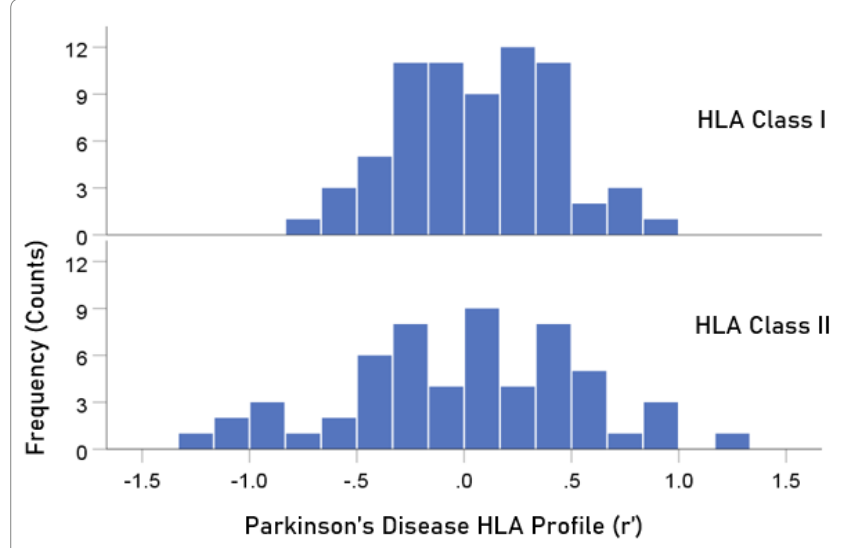

Figure 3. Frequency distribution of HLA profiles for Parkinson's disease. $\mathrm{N}=68$ alleles for Class I and 59 alleles for Class II.

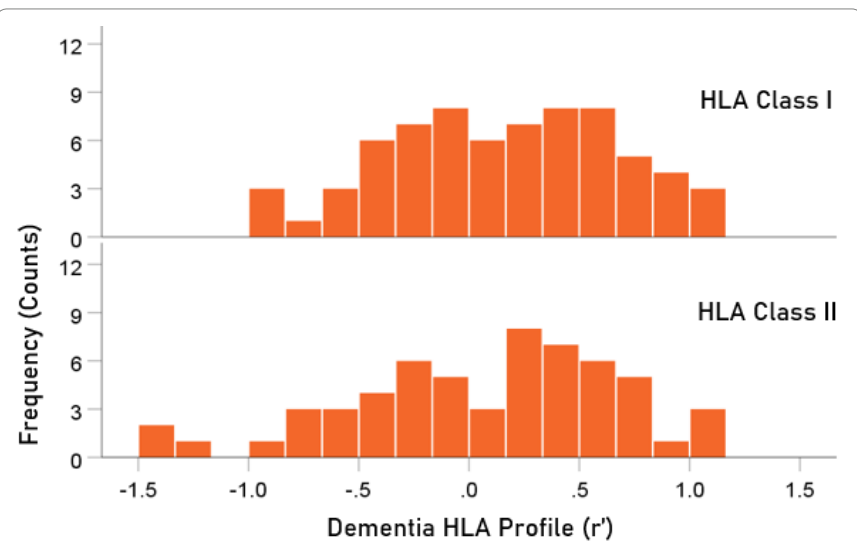

Figure 4. Frequency distribution of HLA profiles for dementia. $\mathrm{N}=68$ alleles for Class I and 59 alleles for Class II.

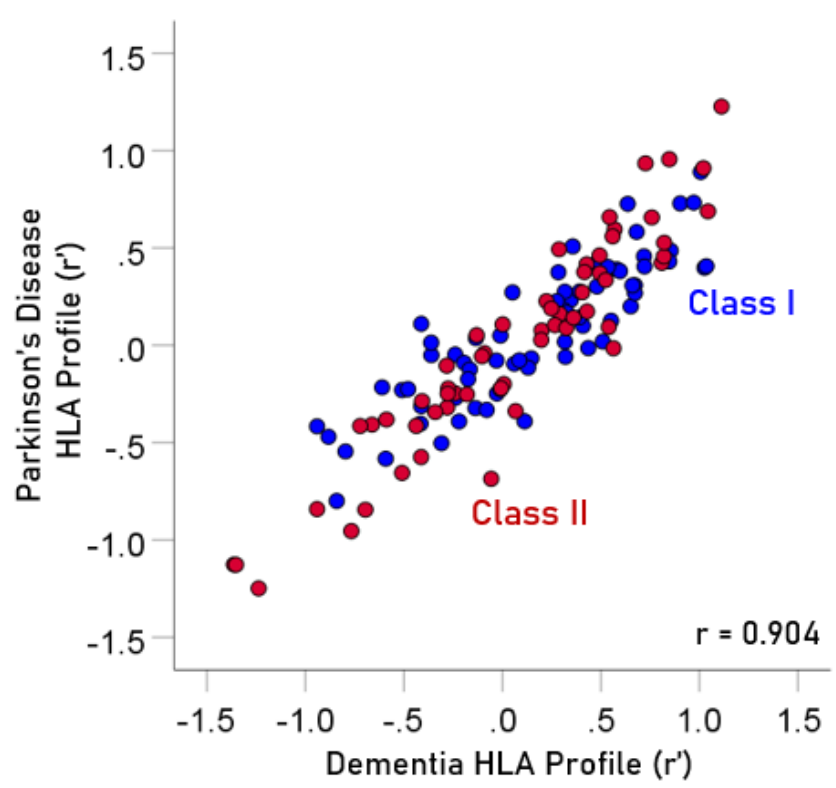

Figure 5. The HLA profile $\left(r^{\prime}\right)$ of Parkinson's disease is plotted against the HLA profile of dementia. The two HLA disease profiles were highly correlated. $\mathrm{N}=127$.

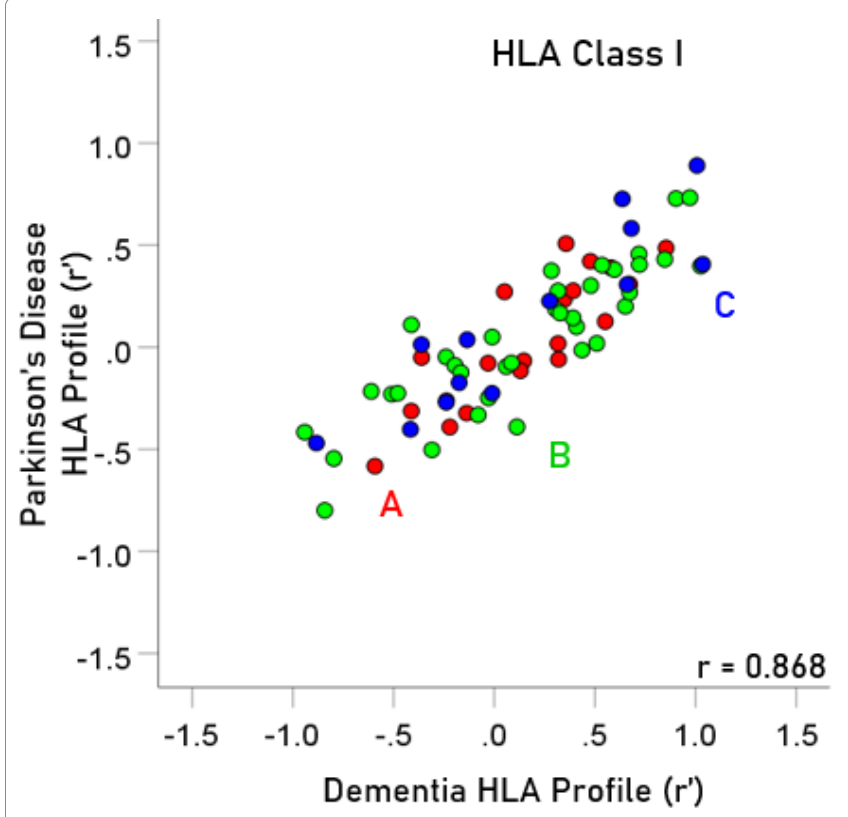

Figure 6. The HLA Class I profile of Parkinson's disease is plotted against the HLA Class I profile of dementia. The two HLA disease profiles were highly correlated. $\mathrm{N}=68$ alleles.

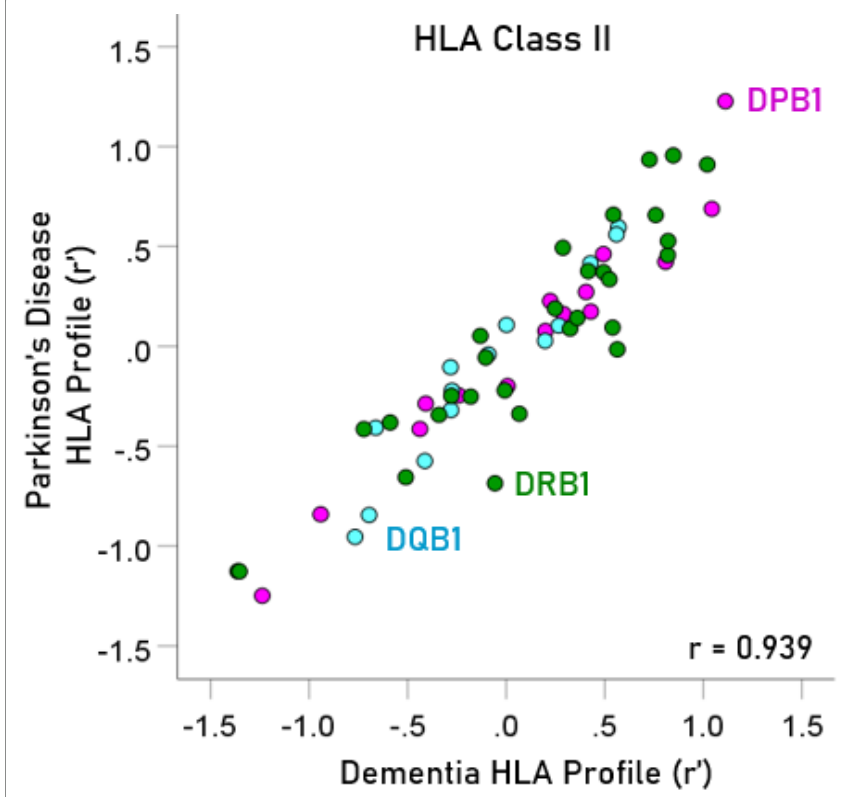

Figure 7. The HLA Class II profile of Parkinson's disease is plotted against the HLA Class II profile of dementia. The two HLA disease profiles were highly correlated. $\mathrm{N}=59$ alleles.

coded in Figure 5). More specifically, the correlation between the two disease HLA profiles was $\mathrm{r}=0.868(\mathrm{P}=$ $\left.4.8 \times 10^{-22}, \mathrm{~N}=69\right)$ for Class I (Figure 6) and $\mathrm{r}=0.939(\mathrm{P}=$ $1.3 \times 10^{-27}, \mathrm{~N}=58$ ) for Class II (Figure 7). In addition, this positive correlation extended across the 3 genes of Class I and the 3 genes of Class II (color coded in Figures 6 and 7, respectively). More specifically, the correlations between disease profiles were as follows: $r=0.854$ (Class I, gene A, 
Table 2: The signed z-transformed correlation coefficient $\left(\boldsymbol{r}^{\prime}\right)$ between 127 HLA alleles and disease prevalence.

\begin{tabular}{|c|c|c|c|c|c|}
\hline & Allele & Class & $\mathbf{N}$ & $r^{\prime}(P D)$ & $r^{\prime}(D E M)$ \\
\hline 1 & $A^{*} 01: 01$ & 1 & 11 & -0.263 & -0.237 \\
\hline 2 & $A * 02: 01$ & 1 & 11 & -0.583 & -0.593 \\
\hline 3 & $A * 02: 05$ & 1 & 9 & 0.486 & 0.853 \\
\hline 4 & $A * 03: 01$ & 1 & 11 & -0.313 & -0.412 \\
\hline 5 & $A^{*} 11: 01$ & 1 & 11 & 0.233 & 0.347 \\
\hline 6 & $A * 23: 01$ & 1 & 11 & 0.018 & 0.317 \\
\hline 7 & $A * 24: 02$ & 1 & 11 & 0.272 & 0.051 \\
\hline 8 & $A * 25: 01$ & 1 & 12 & -0.078 & -0.031 \\
\hline 9 & $A * 26: 01$ & 1 & 11 & 0.421 & 0.478 \\
\hline 10 & $A * 29: 01$ & 1 & 11 & -0.067 & 0.147 \\
\hline 11 & $A * 29: 02$ & 1 & 11 & -0.115 & 0.131 \\
\hline 12 & $A * 30: 01$ & 1 & 11 & 0.508 & 0.357 \\
\hline 13 & $A * 30: 02$ & 1 & 12 & 0.390 & 0.580 \\
\hline 14 & $A * 31: 01$ & 1 & 9 & -0.051 & -0.361 \\
\hline 15 & $A * 32: 01$ & 1 & 12 & 0.277 & 0.392 \\
\hline 16 & $A * 33: 01$ & 1 & 10 & 0.308 & 0.672 \\
\hline 17 & $A * 33: 03$ & 1 & 9 & -0.059 & 0.320 \\
\hline 18 & $A * 36: 01$ & 1 & 10 & 0.126 & 0.551 \\
\hline 19 & $A * 68: 01$ & 1 & 11 & -0.391 & -0.220 \\
\hline 20 & $A * 68: 02$ & 1 & 10 & -0.323 & -0.137 \\
\hline 21 & $B * 07: 02$ & 1 & 10 & -0.416 & -0.941 \\
\hline 22 & $B * 08: 01$ & 1 & 12 & -0.545 & -0.797 \\
\hline 23 & $B * 13: 02$ & 1 & 11 & -0.047 & -0.240 \\
\hline 24 & B*14:01 & 1 & 11 & 0.100 & 0.409 \\
\hline 25 & $B * 14: 02$ & 1 & 10 & 0.457 & 0.719 \\
\hline 26 & $B * 15: 01$ & 1 & 10 & -0.216 & -0.611 \\
\hline 27 & B*15:17 & 1 & 9 & 0.728 & 0.903 \\
\hline 28 & $B * 15: 18$ & 1 & 9 & 0.302 & 0.479 \\
\hline 29 & B*18:01 & 1 & 12 & 0.381 & 0.597 \\
\hline 30 & $B * 27: 02$ & 1 & 10 & -0.095 & 0.060 \\
\hline 31 & $B * 27: 05$ & 1 & 12 & -0.090 & -0.195 \\
\hline 32 & $B * 35: 01$ & 1 & 11 & 0.186 & 0.306 \\
\hline 33 & $B * 35: 02$ & 1 & 9 & 0.200 & 0.651 \\
\hline 34 & $B * 35: 03$ & 1 & 9 & 0.268 & 0.672 \\
\hline 35 & $B * 35: 08$ & 1 & 9 & 0.733 & 0.971 \\
\hline 36 & $B * 37: 01$ & 1 & 10 & 0.110 & -0.412 \\
\hline 37 & $B * 38: 01$ & 1 & 9 & 0.398 & 1.026 \\
\hline 38 & $B * 39: 01$ & 1 & 11 & 0.375 & 0.284 \\
\hline 39 & B*39:06 & 1 & 9 & -0.332 & -0.080 \\
\hline 40 & $B * 40: 01$ & 1 & 12 & -0.230 & -0.509 \\
\hline 41 & $B * 40: 02$ & 1 & 12 & 0.050 & -0.010 \\
\hline 42 & B*41:01 & 1 & 11 & 0.144 & 0.391 \\
\hline 43 & $B * 41: 02$ & 1 & 10 & -0.014 & 0.436 \\
\hline 44 & $B * 44: 02$ & 1 & 12 & -0.799 & -0.841 \\
\hline 45 & $B * 44: 03$ & 1 & 12 & -0.077 & 0.086 \\
\hline 46 & $B * 44: 05$ & 1 & 9 & 0.167 & 0.327 \\
\hline 47 & $B * 45: 01$ & 1 & 10 & -0.248 & -0.028 \\
\hline 48 & $B * 47: 01$ & 1 & 11 & -0.391 & 0.113 \\
\hline 49 & B*49:01 & 1 & 11 & 0.431 & 0.847 \\
\hline 50 & $B * 50: 01$ & 1 & 10 & 0.019 & 0.509 \\
\hline 51 & B*51:01 & 1 & 10 & 0.402 & 0.535 \\
\hline
\end{tabular}

\begin{tabular}{|c|c|c|c|c|c|}
\hline 52 & $B * 52: 01$ & 1 & 10 & 0.276 & 0.317 \\
\hline 53 & $B * 55: 01$ & 1 & 11 & -0.225 & -0.479 \\
\hline 54 & $B * 56: 01$ & 1 & 9 & -0.503 & -0.309 \\
\hline 55 & $B * 57: 01$ & 1 & 12 & -0.124 & -0.166 \\
\hline 56 & $B * 58: 01$ & 1 & 9 & 0.405 & 0.721 \\
\hline 57 & $C^{*} 01: 02$ & 1 & 9 & -0.225 & -0.011 \\
\hline 58 & $C * 03: 03$ & 1 & 9 & -0.403 & -0.416 \\
\hline 59 & $C * 04: 01$ & 1 & 9 & 0.406 & 1.035 \\
\hline 60 & $C * 05: 01$ & 1 & 9 & -0.174 & -0.174 \\
\hline 61 & $C * 06: 02$ & 1 & 9 & 0.037 & -0.135 \\
\hline 62 & $C * 07: 01$ & 1 & 9 & 0.013 & -0.362 \\
\hline 63 & $C^{*} 07: 02$ & 1 & 9 & -0.470 & -0.883 \\
\hline 64 & $C * 07: 04$ & 1 & 9 & -0.270 & -0.238 \\
\hline 65 & $C * 12: 02$ & 1 & 9 & 0.727 & 0.636 \\
\hline 66 & $C * 12: 03$ & 1 & 9 & 0.890 & 1.007 \\
\hline 67 & $C * 14: 02$ & 1 & 9 & 0.582 & 0.681 \\
\hline 68 & C*15:02 & 1 & 9 & 0.307 & 0.660 \\
\hline 69 & $C * 16: 01$ & 1 & 9 & 0.226 & 0.275 \\
\hline 70 & DPB1*01:01 & 2 & 11 & -0.841 & -0.941 \\
\hline 71 & DPB1*02:01 & 2 & 11 & 1.226 & 1.112 \\
\hline 72 & DPB1*02:02 & 2 & 10 & 0.272 & 0.405 \\
\hline 73 & DPB1*03:01 & 2 & 11 & 0.462 & 0.493 \\
\hline 74 & DPB1*04:01 & 2 & 11 & -1.248 & -1.238 \\
\hline 75 & DPB1*04:02 & 2 & 11 & -0.414 & -0.438 \\
\hline 76 & DPB1*05:01 & 2 & 11 & -0.287 & -0.408 \\
\hline 77 & DPB1*06:01 & 2 & 10 & 0.077 & 0.199 \\
\hline 78 & DPB1*09:01 & 2 & 9 & 0.688 & 1.043 \\
\hline 79 & DPB1*10:01 & 2 & 10 & 0.423 & 0.809 \\
\hline 80 & DPB1*11:01 & 2 & 9 & -0.199 & 0.007 \\
\hline 81 & DPB1*13:01 & 2 & 10 & 0.161 & 0.291 \\
\hline 82 & DPB1*14:01 & 2 & 11 & 0.227 & 0.223 \\
\hline 83 & DPB1*17:01 & 2 & 9 & 0.174 & 0.429 \\
\hline 84 & DPB1*19:01 & 2 & 11 & -0.246 & -0.241 \\
\hline 85 & DQB1*02:01 & 2 & 12 & -0.574 & -0.413 \\
\hline 86 & DQB1*02:02 & 2 & 11 & 0.028 & 0.197 \\
\hline 87 & DQB1*03:01 & 2 & 13 & 0.595 & 0.569 \\
\hline 88 & DQB1*03:02 & 2 & 13 & -0.320 & -0.280 \\
\hline 89 & DQB1*03:03 & 2 & 13 & -0.105 & -0.282 \\
\hline 90 & DQB1*04:02 & 2 & 13 & -0.041 & -0.091 \\
\hline 91 & DQB1*05:01 & 2 & 13 & -0.222 & -0.275 \\
\hline 92 & DQB1*05:02 & 2 & 10 & 0.560 & 0.560 \\
\hline 93 & DQB1*05:03 & 2 & 12 & 0.416 & 0.428 \\
\hline 94 & DQB1*06:01 & 2 & 11 & 0.104 & 0.267 \\
\hline 95 & DQB1*06:02 & 2 & 14 & -0.844 & -0.696 \\
\hline 96 & DQB1*06:03 & 2 & 13 & -0.954 & -0.767 \\
\hline 97 & DQB1*06:04 & 2 & 12 & -0.407 & -0.662 \\
\hline 98 & DQB1*06:09 & 2 & 9 & 0.107 & 0.001 \\
\hline 99 & DRB1*01:01 & 2 & 14 & -0.414 & -0.722 \\
\hline 100 & DRB1*01:02 & 2 & 11 & 0.094 & 0.540 \\
\hline 101 & DRB1*01:03 & 2 & 11 & -0.338 & 0.067 \\
\hline 102 & DRB1*03:01 & 2 & 13 & 0.370 & 0.494 \\
\hline 103 & DRB1*04:01 & 2 & 13 & -1.126 & -1.363 \\
\hline 104 & DRB1*04:02 & 2 & 11 & 0.657 & 0.758 \\
\hline 105 & DRB1*04:03 & 2 & 12 & 0.335 & 0.524 \\
\hline 106 & DRB1*04:04 & 2 & 13 & -0.248 & -0.278 \\
\hline
\end{tabular}




\begin{tabular}{|l|l|l|l|l|l|}
\hline 107 & DRB1*04:05 & 2 & 9 & 0.910 & 1.020 \\
\hline 108 & DRB1*04:07 & 2 & 12 & 0.189 & 0.248 \\
\hline 109 & DRB1*04:08 & 2 & 9 & -0.343 & -0.341 \\
\hline 110 & DRB1*07:01 & 2 & 12 & -0.221 & -0.007 \\
\hline 111 & DRB1*08:01 & 2 & 13 & -0.056 & -0.104 \\
\hline 112 & DRB1*08:03 & 2 & 11 & -0.686 & -0.057 \\
\hline 113 & DRB1*09:01 & 2 & 12 & 0.052 & -0.131 \\
\hline 114 & DRB1*10:01 & 2 & 14 & 0.493 & 0.288 \\
\hline 115 & DRB1*11:01 & 2 & 14 & 0.956 & 0.847 \\
\hline 116 & DRB1*11:02 & 2 & 12 & -0.015 & 0.564 \\
\hline 117 & DRB1*11:03 & 2 & 12 & 0.456 & 0.820 \\
\hline 118 & DRB1*11:04 & 2 & 12 & 0.934 & 0.726 \\
\hline 119 & DRB1*12:01 & 2 & 13 & -0.252 & -0.180 \\
\hline 120 & DRB1*13:01 & 2 & 14 & -0.656 & -0.509 \\
\hline 121 & DRB1*13:02 & 2 & 14 & -0.382 & -0.589 \\
\hline 122 & DRB1*13:03 & 2 & 10 & 0.527 & 0.821 \\
\hline 123 & DRB1*13:05 & 2 & 10 & 0.088 & 0.324 \\
\hline 124 & DRB1*14:01 & 2 & 14 & 0.142 & 0.361 \\
\hline 125 & DRB1*15:01 & 2 & 13 & -1.127 & -1.353 \\
\hline 126 & DRB1*15:02 & 2 & 10 & 0.376 & 0.416 \\
\hline 127 & DRB1*16:01 & 2 & 10 & 0.659 & 0.544 \\
\hline & & & & & \\
\hline
\end{tabular}

Table 3: Two-way table of the distribution of signed correlation counts in the whole sample ( $\mathrm{N}=127$ alleles).

\begin{tabular}{|c|c|c|c|c|}
\hline & \multicolumn{2}{|c|}{ Dementia } & \multirow[b]{2}{*}{ Total } \\
\hline & & Negative & Positive & \\
\hline \multirow{3}{*}{ Parkinson's disease } & Negative & 48 & 10 & 58 \\
\hline & Positive & 5 & 64 & 69 \\
\hline & Total & 53 & 74 & 127 \\
\hline
\end{tabular}

Table 4: Two-way table of the distribution of signed correlation counts in HLA Class I ( $\mathrm{N}=69$ alleles).

\begin{tabular}{|l|c|c|c|c|}
\hline \multicolumn{2}{|c|}{} & \multicolumn{2}{c|}{ Dementia } & \\
\cline { 3 - 5 } \multicolumn{2}{|c|}{} & Negative & Positive & Total \\
\hline \multirow{2}{*}{ Parkinson's disease } & Negative & 24 & 7 & 31 \\
\cline { 2 - 5 } & Positive & 4 & 34 & 38 \\
\hline & Total & 28 & 41 & 69 \\
\hline
\end{tabular}

$\mathrm{P}=2.0 \times 10^{-6}, \mathrm{~N}=20$ ), 0.867 (Class I, gene $\mathrm{B}, \mathrm{P}=7.8 \times 10^{-12}$, $\mathrm{N}=30$ ), 0.903 (Class I, gene $\mathrm{C}, \mathrm{P}=2.3 \times 10^{-5}, \mathrm{~N}=13$ ), 0.974 (Class II, gene DPB1, P = $9.5 \times 10^{-10}, \mathrm{~N}=15$ ), 0.957 (Class II, gene DQB1, $\mathrm{P}=8.8 \times 10^{-8}, \mathrm{~N}=14$ ) 0.914 (Class II, gene DRB1, $\mathrm{P}=4.1 \times 10^{-12}, \mathrm{~N}=29$ ).

\section{Analysis of counts of signed correlations}

The two-way distribution of the counts of signed correlations between allele frequency and disease prevalence is given in Table 3 . There was a highly significant positive association (chi-square $=73.89, \mathrm{P}(2$-sided $)=8.2$ $\mathrm{x} 10^{-18}$; Fisher's exact test (2-sided) $\mathrm{P}=4.4 \times 10^{-19}$; phi $=$ $0.763)$.

This positive association was present in both HLA Class I (Table 4) and Class II (Table 5). For Class I: chi-square = 31.68, $\mathrm{P}(2$-sided $)=1.8 \times 10^{-8}$; Fisher's exact test $(2$-sided $)$
Table 5: Two-way table of the distribution of signed correlation counts in HLA Class II ( $\mathrm{N}=58$ alleles).

\begin{tabular}{|l|c|c|c|c|}
\hline \multicolumn{2}{|c|}{} & \multicolumn{2}{c|}{ Dementia } & \\
\cline { 3 - 5 } & Negative & Positive & Total \\
\hline \multirow{2}{*}{ Parkinson's disease } & Negative & 24 & 3 & 27 \\
\cline { 2 - 5 } & Positive & 1 & 30 & 31 \\
\hline & Total & 25 & 33 & 58 \\
\hline
\end{tabular}

Table 6: Two-way tables of the distribution of signed correlation counts in all six HLA Class I and II alleles. Values in parentheses are two-sided probability values of the Fisher's exact test.

\begin{tabular}{|c|c|c|c|c|c|c|}
\hline & & & & Dementi & & \\
\hline & & & & Negative & Positive & Total \\
\hline & & & Negative & 7 & 3 & 10 \\
\hline & & A & Positive & 0 & 10 & 10 \\
\hline & & & Total & 7 & 13 & 20 \\
\hline & & & Negative & 12 & 4 & 16 \\
\hline & Class I & B & Positive & 2 & 18 & 20 \\
\hline & & & Total & 14 & 22 & 36 \\
\hline & & & Negative & 5 & 0 & 5 \\
\hline & & $C$ & Positive & 2 & 6 & 8 \\
\hline Parkinson's & & & Total & 5 & 6 & 13 \\
\hline disease & & & Negative & 5 & 1 & 6 \\
\hline & & $\begin{array}{l}\text { DPB1 } \\
(P=020)\end{array}$ & Positive & 0 & 9 & 9 \\
\hline & & & Total & 5 & 10 & 15 \\
\hline & & & Negative & 8 & 0 & 8 \\
\hline & Class II & $\begin{array}{l}\text { DUB1 } \\
(P=0,0003)\end{array}$ & Positive & 0 & 6 & 6 \\
\hline & & & Total & 8 & 6 & 14 \\
\hline & & & Negative & 11 & 2 & 13 \\
\hline & & $\begin{array}{l}D K B \perp \\
(P=0,0002)\end{array}$ & Positive & 1 & 15 & 16 \\
\hline & & & Total & 12 & 17 & 29 \\
\hline
\end{tabular}

$\mathrm{P}=1.4 \times 10^{-8}$; phi $\left.=0.678\right)$. For Class II, this association was stronger: chi-square $=43.18, \mathrm{P}(2$-sided $)=5.0 \times 10^{-11}$; Fisher's exact test (2-sided) $\mathrm{P}=5.2 \times 10^{-12}$; phi $=0.863$ ). Similar results were obtained for each one of the six alleles (Table 6).

\section{Comparison of Parkinson's disease and dementia with respect to protective and susceptibility alleles}

Protective alleles. There were 48 alleles protective for both diseases. The magnitude of $r^{\prime}$ did not differ significantly between the two diseases $(\mathrm{P}=0.221$, paired t-test); for Parkinson's disease, $r^{\prime}$ (mean \pm SEM) was $-0.403 \pm 0.043$, and for dementia $-0.440 \pm 0.050$. Very similar results were obtained when the data were analyzed separately by a paired t-test for Class I and Class II alleles: for Class I, $\mathrm{P}=0.156, \mathrm{~N}=24$, and for Class II, $\mathrm{P}=0.824, \mathrm{~N}$ = 24).

Susceptibility alleles. There were 64 susceptibility alleles for both diseases. The magnitude of $r^{\prime}$ was significantly higher for dementia than for Parkinson's disease ( $\mathrm{P}=7.9$ $\mathrm{x} 10^{-9}$, paired t-test); for Parkinson's disease, $r^{\prime}$ (mean \pm $\mathrm{SEM}$ ) was $0.338 \pm 0.032$, and for dementia $0.552 \pm 0.033$. 
Table 7: Correlation coefficients between $r^{\prime}(\boldsymbol{P D})$ and $r^{\prime}(\boldsymbol{D E M})$ at various levels of numbers of alleles available in the 14 CWE countries.

\begin{tabular}{|c|c|c|c|}
\hline $\begin{array}{c}\text { Number of } \\
\text { countries with N } \\
\text { alleles }\end{array}$ & $\begin{array}{c}\text { Number of } \\
\text { distinct alleles }\end{array}$ & $\begin{array}{c}\text { Correlation between } \\
r^{\prime}(P D) \text { and } r^{\prime}(D E M)\end{array}$ & $\mathbf{P}$-value \\
\hline$\geq 3$ & 266 & 0.748 & $\mathrm{P}<0.0001$ \\
\hline$\geq 4$ & 217 & 0.798 & $\mathrm{P}<0.0001$ \\
\hline$\geq 5$ & 186 & 0.852 & $\mathrm{P}<0.0001$ \\
\hline$\geq 6$ & 171 & 0.877 & $\mathrm{P}<0.0001$ \\
\hline$\geq 7$ & 162 & 0.878 & $\mathrm{P}<0.0001$ \\
\hline$\geq 8$ & 151 & 0.884 & $\mathrm{P}<0.0001$ \\
\hline$\geq 9$ & 127 & 0.904 & $\mathrm{P}<0.0001$ \\
\hline$\geq 10$ & 95 & 0.903 & $\mathrm{P}<0.0001$ \\
\hline$\geq 11$ & 73 & 0.920 & $\mathrm{P}<0.0001$ \\
\hline$\geq 12$ & 40 & 0.941 & $\mathrm{P}<0.0001$ \\
\hline$\geq 13$ & 19 & 0.964 & $\mathrm{P}<0.0001$ \\
\hline 14 & 7 & 0.946 & $\mathrm{P}=0.0012$ \\
\hline & & & \\
\hline
\end{tabular}

Very similar results were obtained when the data were analyzed separately by a paired t-test for Class I and Class II alleles: for Class I, $\mathrm{P}=4.1 \times 10^{-7}, \mathrm{~N}=34$, and for Class II, $\mathrm{P}=0.003, \mathrm{~N}=30$ ).

\section{Analyses with different sample sizes}

For the analyses above, we used a minimum sample size of 9 countries as a reasonable choice of sample size. However, we also computed HLA profiles for all available samples sizes $\geq 3$ and calculated correlations between these PD and dementia HLA profiles to check for consistency of their correlation. The results are shown in Table 7. It can be seen that a highly significant positive correlation between the 2 disease profiles was obtained for all cases of $\mathrm{N} \geq 3$.

\section{Discussion}

Here we used an across-countries immunogenetic epidemiological approach ${ }^{22}$ to identify HLA profiles for dementia and Parkinson's disease and evaluate their correspondence using data obtained from 14 countries in Continental Western Europe. The results demonstrated that the HLA profiles of the two diseases are remarkably similar when examined in aggregate and separately for each of the Class I and Class II alleles. However, when considered with regard to HLA protection or susceptibility, the correlation of susceptibility HLA alleles with dementia was significantly stronger than their correlation with Parkinson's disease, whereas there was no significant difference regarding the correlation of protective/preventive alleles with dementia or Parkinson's disease. These findings extend previous research demonstrating highly similar HLA DRB1 profiles in dementia and Parkinson's disease ${ }^{3}$ to a large number of other Class I and Class II HLA alleles and point to increased immunogenetic susceptibility to dementia relative to Parkinson's disease. Our view of how HLA could be involved in prevention/protection from, and susceptibility to, these

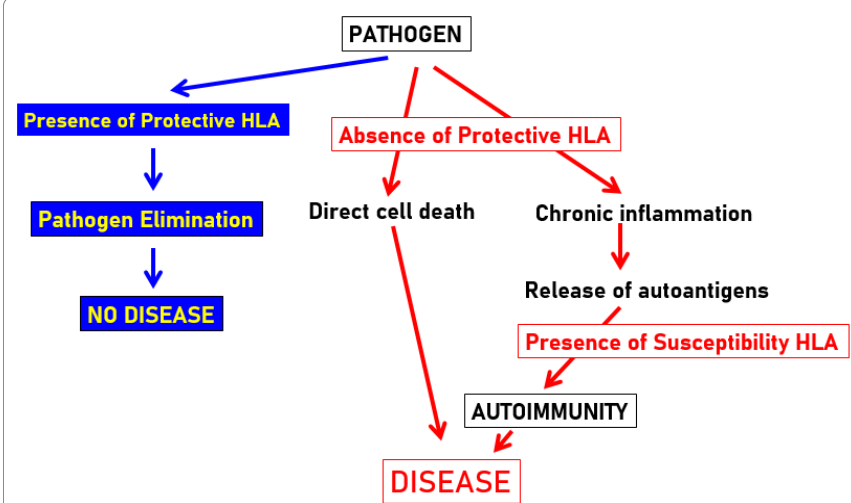

Figure 8. Diagram illustrating putative protective and susceptibility roles of HLA in disease outcomes.

and other diseases is discussed below and exemplified in the schematic diagram of Figure 8.

It is well-established that the HLA system evolved for, and is explicitly involved in, pathogen elimination. Thus, with respect to protective effects identified here, we assume that protective HLA alleles exert their effects via elimination of pathogens, thereby preventing deleterious downstream health effects. Our finding that protective HLA profiles (i.e., alleles with negative $r^{\prime}$ ) did not differ significantly between the two diseases is consistent with evidence implicating similar families of pathogens in both Parkinson's disease and dementia ${ }^{6-8,27-34}$.

In the absence of HLA protection against pathogens, disease may result from directly damaging effects of a pathogen on cells or as a result of susceptibility HLA alleles (i.e., alleles with positive $r^{\prime}$ ) that promote autoimmunity (i.e., production of autoantibodies) due to chronic inflammation ${ }^{16,35}$. This is in accord with evidence documenting inflammatory and autoimmune processes in both dementia and Parkinson's disease ${ }^{9,27,36}$ as well as evidence of autoantibodies in both conditions ${ }^{37-41}$. Here, susceptibility (i.e., positively associated) alleles occurred more frequently than protective (i.e., negatively associated) alleles, indicating a preponderance of HLA alleles that may increase susceptibility to autoimmunity.

At the individual level, HLA composition plays a critical role in influencing health vs immune-mediated disease outcomes due to the protective role of HLA in eliminating foreign antigens ${ }^{42}$ and to its predisposing role in autoimmunity ${ }^{16}$. As previously noted, HLA is the most highly polymorphic region of the human genome. This is notable in that subtle alterations in HLA have been shown to affect the binding groove, resulting in differential binding affinity of antigens ${ }^{43,44}$. We have hypothesized that protection against diseases including dementia and Parkinson's disease conferred by specific HLA alleles is related to their superior ability to bind, and therefore eliminate, harmful antigens. On the other hand, inability to 
bind antigens and mount an immune response is posited to result in persistent antigens that may directly damage cells and/or may potentially stimulate chronic inflammatory responses and/or autoimmunity ${ }^{45-47}$ and ultimately clinical disease ${ }^{42}$. Dementia commonly occurs in individuals with Parkinson's disease ${ }^{48}$. The extent to which the cooccurrence may be a result of pathogen-driven cell death or autoimmunity is unclear.

It is worth noting that the persistent antigen hypothesis is complementary rather than inconsistent with prevailing theories implicating aggregated self-proteins in dementia and Parkinson's disease. Indeed, both amyloid- $\beta$ and alpha-synuclein, proteins associated with dementia and Parkinson's disease, respectively, have been shown to exhibit anti-microbial properties ${ }^{29,49,50}$. That is, growing research suggests that aggregated proteins that have long been characterized as the hallmark pathological characteristics of neurodegenerative disorders may initially reflect a protective immune response to infections ${ }^{29,49,50}$. If, however, foreign antigens persist due to lack of HLAantigen congruence as suggested by the persistent antigen hypothesis ${ }^{42}$, the amyloid- $\beta$ and/or alpha-synuclein protein aggregation may continue, resulting in unchecked protein deposition. In turn, aggregated self-proteins may be recognized as foreign antigens, stimulating further immune system reactivity ${ }^{47}$.

Here we found no differences between dementia and Parkinson's disease prevalence with respect to protective HLA alleles for both conditions; however, the association of susceptibility HLA alleles was stronger with dementia than the association of those same alleles with Parkinson's disease. These findings suggest that similar immunogenetic mechanisms along the lines of those discussed above (i.e., elimination of pathogens) are involved in protection against these conditions but that additional factors are involved in moderating HLA-mediated autoimmunity in dementia and Parkinson's disease, including variations in apo $E^{51,52}$, modifiable risk factors including diet, exercise, and smoking, among others $\mathrm{s}^{4,5,53}$, and other environmental exposures that have been differentially associated with dementia and Parkinson's disease ${ }^{4,5}$. These issues represent important areas for future investigation.

A broad link between HLA and neurodegenerative diseases such as dementia and Parkinson's disease has been increasingly recognized ${ }^{1-3,9,17-21}$; however, the influence of specific genes on these diseases has not been clearly established and findings have often been inconsistent. For example, we have identified population-level protective effects of DRB1*15:01 on dementia and Parkinson's disease here, and have previously shown that DRB1*15:01 binds with viruses linked to dementia and Parkinson's disease with very high affinity ${ }^{54}$. In contrast, another recent study identified DRB1*15:01 as a risk factor for Alzheimer's dementia ${ }^{19}$; however, in that study HLA was imputed rather than directly sequenced, and the findings regarding DRB1*15:01 were limited to men lacking the ApoE4 risk gene. Thus, additional research using direct sequencing of HLA is warranted to further evaluate the association of HLA alleles, including DRB1*15:01, to dementia and to evaluate the effects of gender and other moderating factors. Similarly, although the findings regarding associations between HLA (including DRB1*15:01) and Parkinson's disease are more compelling ${ }^{55-57}$, additional research is warranted to more conclusively establish HLA associations with Parkinson's disease and to evaluate variations in HLA-disease associations across different populations given global variations in HLA. Determining specific HLA influences on diseases move beyond the common influence of inflammation in dementia and Parkinson's disease to facilitate in silico analyses that permit identification of pathogen families that may contribute to inflammation and disease (e.g., ref $^{54}$ ).

\section{Limitations and qualifications}

There are several limitations/qualifications of this study, as follows. First, these results are based on correlations between disease prevalences and HLA allele frequencies in large populations of $14 \mathrm{CWE}$ countries and, as such, they need to be validated in studies using assessments of disease and allele presence in specific individuals. Second, it is known that HLA-disease associations may vary from place to place ${ }^{58,59}$, and across countries ${ }^{22,59}$ and, therefore, the results of this study are properly applicable to the $14 \mathrm{CWE}$ countries used here but could be extended to other countries/regions with further analyses, as done for malaria ${ }^{22}$. Finally, it should be noted that the dementia population(s) in the GBD study used here ${ }^{4}$ include dementia cases from Parkinson's disease. However, the estimated percentage of dementia attributable to Parkinson's disease in the population is only $3-4 \%$ globally ${ }^{60}$, and reducing the GBD dementia population counts accordingly would yield the same correlations between the adjusted dementia prevalence and HLA allele frequency, since the percentage above is generally consistent from country to country.

\section{Summary and Conclusions}

The present study documents highly overlapping HLA profiles for dementia and Parkinson's disease at the population level in Continental Western Europe, suggesting that similar population-level immunogenetic mechanisms contribute to prevention and/or susceptibility to both conditions. With respect to prevention, HLA plays a major role in the elimination of foreign antigens and, hence, its protective role found here can be attributed to the elimination of potential pathogens implicated as putative causative agents for these diseases (e.g., human herpes viruses ${ }^{31}$ ). HLA also 
plays a major role in autoimmunity which may occur in the absence of protection and in the presence of ensuing chronic inflammation. In both protection (pathogen elimination) and susceptibility (autoimmunity) cases, the HLA overlap between dementia and Parkinson's disease indicates a similarity/overlap in the family of putative pathogens and autoantigens, respectively. The identification of such pathogens and autoantigens could be further investigated (e.g., in silico) using information from the specific alleles involved in protection and susceptibility. Finally, the present findings and future prospects regarding identification of disease-associated pathogens and autoantigens based on a disease's HLAprofile extend beyond common neurodegenerative diseases to include a wide range of conditions for which pathogens and/or autoimmunity have been implicated, both in specific regions and globally.

\section{Abbreviations}

\section{HLA: Human Leukocyte Antigen}

\section{Author contributions}

APG contributed to data analysis; LMJ and APG contributed to writing the manuscript.

\section{Conflicts of Interest}

The authors declare that the research was conducted in the absence of any commercial or financial relationships that could be construed as a potential conflict of interest.

\section{Ethical Approval}

The research was approved by the Minneapolis VA Health Care System Research and Development Committee (Reference Number 006265).

\section{Consent to Participate}

Not applicable.

\section{Consent to Publication}

Not applicable.

\section{Availability of Data and Materials}

The datasets analyzed for this study are publicly available. The data can be found in the Allele Frequency Net Database (allelefrequencies.net) and in publications ${ }^{4,5,23}$.

\section{Funding}

Partial funding for this study was provided by the University of Minnesota (the Anita Kunin Chair in Women's Healthy Brain Aging, the Brain and Genomics Fund, the McKnight Presidential Chair of Cognitive Neuroscience, and the American Legion Brain Sciences Chair) and the U.S. Department of Veterans Affairs. The sponsors had no role in the current study design, analysis or interpretation, or in the writing of this paper. The contents do not represent the views of the U.S. Department of Veterans Affairs or the United States Government.

\section{References}

1. James LM, Georgopoulos AP. The human leukocyte antigen (HLA) DRB1*13:02 allele protects against dementia in continental Western Europe. J Neurol Neuromed. 2019; 4(5): 1-6.

2. James LM, Georgopoulos AP. Tri-Allelic Human Leukocyte Antigen (HLA) Protection Against Dementia. J Neurol Neuromed. 2020; 5(1): 12-17.

3. James LM, Georgopoulos AP. Shared Human Leukocyte Antigen (HLA) Coverage in Dementia and Parkinson's disease. J Neurol Neuromed. 2020; 5(3): 45-54.

4. GBD 2016 Dementia Collaborators. Global, regional, and national burden of Alzheimer's disease and other dementias, 1990-2016: a systematic analysis for the Global Burden of Disease Study 2016. Lancet Neurol. 2019; 18(1): 88-106.

5. GBD 2016 Parkinson's Disease Collaborators. Global, regional, and national burden of Parkinson's disease, 1990-2016: a systematic analysis for the Global Burden of Disease Study 2016. Lancet Neurol. 2018; 17(11): 939-53.

6. Limphaibool N, Iwanowski P, Holstad MJV, et al. Infectious etiologies of Parkinsonism: Pathomechanisms and clinical implications. Front Neurol. 2019; 10: 652.

7. Almeida OP, Lautenschlager NT. Dementia associated with infectious diseases. Int Psychogeriatr. 2005; 17(Suppl 1): S65-77.

8. Sochocka M, Zwolińska K, Leszek J. The infectious etiology of Alzheimer's Disease. Curr Neuropharmacol. 2017; 15(7): 996-1009.

9. Lindestam Arlehamn CS, Garretti F, Sulzer D, et al. Roles for the adaptive immune system in Parkinson's and Alzheimer's diseases. Curr Opin Immunol. 2019; 59: 115-120.

10. Heppner FL, Ransohoff RM, Becher B. Immune attack: the role of inflammation in Alzheimer disease. Nat Rev Neurosci. 2015; 16(6): 358-72.

11. Tansey MG, Romero-Ramos M. Immune system responses in Parkinson's disease: Early and dynamic. Eur Jour Neurosci. 2019; 49(3): 364-83.

12. van der Willik KD, Fani L, Rizopoulos D, et al. Balance between innate versus adaptive immune system and the risk of dementia: a population-based cohort study. J Neuroinflamm. 2019; 16: 68.

13. Kannarkat GT, Boss JM, Tansey MG. The role of innate and adaptive immunity in Parkinson's disease. J Parkinson Dis. 2013; 3(4): 493514.

14. Sanchez-Mazas A, Lemaître JF, Currat M. Distinct evolutionary strategies of human leucocyte antigen loci in pathogen-rich environments. Philos Trans R Soc Lond B Biol Sci. 2012; 367(1590): 830-9.

15. Salamon H, Klitz W, Easteal S, et al. Evolution of HLA class II molecules: Allelic and amino acid site variability across populations. Genetics. 1999; 152(1): 393-400.

16. Simmonds MJ, Gough SC. The HLA region and autoimmune disease: Associations and mechanisms of action. Curr Genomics. 2007; 8(7): 453-65.

17. Aliseychik MP, Andreeva TV, Rogaev EI. Immunogenetic Factors of Neurodegenerative Diseases: The Role of HLA Class II. Biochemistry Moscow. 2018; 83: 1104-1116.

18. Lambert JC, Ibrahim-Verbaas CA, Harold D, et al. Meta-analysis of 74,046 individuals identifies 11 new susceptibility loci for Alzheimer's disease. Nat Genet. 2013; 45(12): 1452-1458. doi:10.1038/ng.2802 1 
19. Steele NZ, Carr JS, Bonham LW, et al. Fine-mapping of the human leukocyte antigen locus as a risk factor for Alzheimer disease: a case- control study. PLOS Med. 2017; 14(3): e1002272. doi:10.1371/ journal.pmed.1002272.

20. Wang ZX, Wan Q Xing A. HLA in Alzheimer's Disease: Genetic Association and Possible Pathogenic Roles. Neuromol Med. 2020; 22: 464-473.

21. Nalls MA, Blauwendraat C, Vallerga CL, et al. Identification of novel risk loci, causal insights, and heritable risk for Parkinson's disease: a meta-analysis of genome-wide association studies. Lancet Neurol. 2019; 18(12): 1091-102.

22. Garamszegi LZ. Global distribution of malaria-resistant MHC-HLA alleles: the number and frequencies of alleles and malaria risk. Malar J. 2014; 13: 349. doi: 10.1186/1475-2875-13-349.

23. Population Reference Bureau. 2016 world population data sheet with a special focus on human needs and sustainable resources. Population Reference Bureau, Washington, DC, 2016. https://www. prb.org/2016-world-population-data-sheet/. Accessed February 5, 2019.

24. Gonzalez-Galarza FF, Christmas S, Middleton D, et al. Allele frequency net: a database and online repository for immune gene frequencies in worldwide populations. Nucleic Acid Res 2011, 39: D913-D919.

25. Allele*Frequencies in Worldwide Populations [Internet]. Allele frequency net database (AFND) 2020 update. Liverpool, UK. Available from: http://allelefrequencies.net/hla6006a.asp.

26. Fisher RA. Statistical Methods for Research Workers. 13th ed. Edinburgh, Scotland: Oliver \& Boyd; 1958.

27. Caggiu E, Arru G, Hosseini S, et al. Inflammation, infectious triggers, and Parkinson's disease. Front Neurol. 2019; 10: 122

28. Itzhaki RF. Corroboration of a major role for herpes simplex virus type 1 in Alzheimer's disease. Front Aging Neurosci. 2018; 10: e00324.

29. Eimer WA, Kumar DK, Shanmugam NK, et al. Alzheimer's diseaseassociated $\beta$-amyloid is rapidly seeded by herpesviridae to protect against brain infection. Neuron. 2018; 99(1): 56-63.

30. Bu XL, Wang X, Xiang Y, et al. The association between infectious burden and Parkinson's disease: A case-control study. Parkinsonism Relat Dis. 2015; 21: 877-881.

31. Hemling N, Röyttä M, Rinne J, et al. Herpesviruses in brains in Alzheimer's and Parkinson's diseases. Ann Neurol. 2003; 54: 267-271.

32. McGeer PL, McGeer EG. Inflammation and neurodegeneration in Parkinson's disease. Parkinsonism Relat Disord. 2004; 10: S3-7.

33. Wang H, Liu X, Tan C. Bacterial, viral, and fungal infection-related risk of Parkinson's disease: Meta-analysis of cohort and case-control studies. Brain Behav. 2020; 10(3): e01549.

34. Mawanda F, Wallace R. Can infections cause Alzheimer's disease? Epidemiol Rev. 2013; 35: 161-80.

35. Fainboim L, Cañero Velasco MC, Marcos CY, et al. Protracted, but not acute, hepatitis A virus infection is strongly associated with HLADRB*1301, a marker for pediatric autoimmune hepatitis. Hepatology. 2001; 33(6): 1512-7.

36. Sardi F, Fassina L, Venturini L, et al. Alzheimer's disease, autoimmunity and inflammation. The good, the bad and the ugly. Autoimmunity Rev. 2011; 11(2): 149-53.

37. Benkler M, Agmon-Levin N, Hassin-Baer S, et al. Immunology, autoimmunity, and autoantibodies in Parkinson's disease. Clin Rev Allerg Immu. 2012; 42(2): 164-71.

38. Han M, Nagele E, DeMarshall C, et al. Diagnosis of Parkinson's disease based on disease-specific autoantibody profiles in human sera. PloS one. 2012; 7(2): e32383.
39. Shalash A, Salama M, Makar M, Ret al. Elevated serum $\alpha$-synuclein autoantibodies in patients with parkinson's disease relative to alzheimer's disease and controls. Front Neurol. 2017; 8: 720.

40. Nagele E, Han M, DeMarshall C, et al. Diagnosis of Alzheimer's disease based on disease-specific autoantibody profiles in human sera. PloS one. 2011; 6(8): e23112.

41. Wu J, Li L. Autoantibodies in Alzheimer's disease: potential biomarkers, pathogenic roles, and therapeutic implications. J Biomed Res. 2016; 30(5): 361.

42. James LM, Georgopoulos AP. Persistent antigens hypothesis: the human leukocyte antigen (HLA) connection. J Neurol Neuromed. 2018; 3: 27-31.

43. Hov JR, Kosmoliaptsis V, Traherne JA, et al. Electrostatic modifications of the HLA-DR P9 peptide-binding pocket and susceptibility to primary sclerosing cholangitis. Hepatology. 2011; 53: 1967-1976.

44. Davenport MP, Quinn CL, Chicz RM, et al. Naturally processed peptides from two disease-resistance-associated HLA-DR13 alleles show related sequence motifs and the effects of the dimorphism at position 86 of the HLA-DR beta chain. PNAS 1995; 92 (14): 6567-6571.

45. Cusick MF, Libbey JE, Fujinami RS. Molecular mimicry as a mechanism of autoimmune disease. Clin Rev Allergy Immu. 2012; 42(1): 102-11.

46. Severini C, Barbato C, Di Certo MG, et al. Alzheimer's disease: new concepts on the role of autoimmunity and of NLRP3 inflammasome in the pathogenesis of the disease. Curr Neuropharmacol. 2020; 18: 00.

47. Garretti F, Agalliu D, Lindestam Arlehamn CS, et al. Autoimmunity in Parkinson's Disease: the role of $\alpha$-synuclein-specific T cells. Front Immunol. 2019; 10: 303.

48. Emre M, Aarsland D, Brown R, et al. Clinical diagnostic criteria for dementia associated with Parkinson's disease. Mov Disord 2007; 22: 1689-707.

49. Soscia SJ, Kirby JE, Washicosky KJ, et al. The Alzheimer's DiseaseAssociated Amyloid $\beta$-Protein Is an Antimicrobial Peptide. PLoS ONE. 2010; 5(3): e9505.

50. Park SC, Moon JC, Shin SY, et al. Functional characterization of alphasynuclein protein with antimicrobial activity. Biochem Bioph Res Co. 2016; 478(2): 924-8.

51. Corder EH, Saunders AM, Risch NJ, et al. Protective effect of apolipoprotein E type 2 allele for late onset Alzheimer disease. Nat Genet. 1994; $7:$ 180-184.

52. James LM, Georgopoulos AP. Human leukocyte antigen as a key factor in preventing dementia and associated apolipoprotein E4 risk. Front Aging Neurosci. 2019; 11: e82

53. Livingston G, Sommerland A, Orgeta V, et al. Dementia prevention, intervention, and care. Lancet. 2017; 390(10113): 2673-2734.

54. Charonis S, James LM, Georgopoulos AP. In silico assessment of binding affinities of three dementia-protective Human Leukocyte Antigen (HLA) alleles to nine human herpes virus antigens. Curr Res Transl Med. 2020; 68(4): 211-6.

55. Hollenbach JA, Norman PJ, Creary LE, et al. A specific amino acid motif of HLA-DRB1 mediates risk and interacts with smoking history in Parkinson's disease. Proceedings of the National Academy of Sciences. 2019; 116(15): 7419-24.

56. Sulzer D, Alcalay R, Garretti F, et al. T cells from patients with Parkinson's disease recognize $\alpha$-synuclein peptides. Nature. 2017; 546: 656-661.

57. Sun C, Wei L, Luo F, et al. HLA-DRB1 alleles are associated with the susceptibility to sporadic Parkinson's disease in Chinese Han population. PLoS One. 2012; 7(11): e48594.

58. Hill AV, Allsopp CE, Kwiatkowski D, et al. Common west African HLA antigens are associated with protection from severe malaria. Nature. 1991; 352: 595-600. 
59. Hill AV, Yates SN, Allsopp CE, et al. Human leukocyte antigens and natural selection by malaria. Philos Trans R Soc Lond B Biol Sci. 1994; 346: 379-385.
60. Aarsland D, Zaccai J, Brayne C. A systematic review of prevalence studies of dementia in Parkinson's disease. Movement disorders $2005 ; 20: 1255-1263$ 